\title{
Towards next generation 3D cameras
}

\section{Mohit Gupta}

Mohit Gupta, "Towards next generation 3D cameras," Proc. SPIE 10338, Thirteenth International Conference on Quality Control by Artificial Vision 2017, 1033806 (14 May 2017); doi: 10.1117/12.2277465

SPIE Event: The International Conference on Quality Control by Artificial Vision 2017, 2017, Tokyo, Japan 


\section{Plenary Talk I}

\section{Towards Next Generation 3D Cameras}

\section{Prof. Mohit Gupta}

Univ. of Wisconsin-Madison, USA

\section{ABSTRACT}

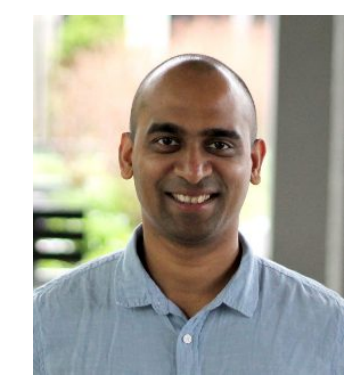

We are in the midst of a $3 D$ revolution. Robots enabled by $3 D$ cameras are beginning to autonomously drive cars, perform surgeries, and manage factories. However, when deployed in the real-world, these cameras face several challenges that prevent them from measuring 3D shape reliably. These challenges include large lighting variations (bright sunlight to dark night), presence of scattering media (fog, body tissue), and optically complex materials (metal, plastic). Due to these factors, 3D imaging is often the bottleneck in widespread adoption of several key robotics technologies.

I will talk about our work on developing 3D cameras based on time-of-flight and active triangulation that addresses these long-standing problems. This includes designing 'all-weather' cameras that can perform high-speed 3D scanning in harsh outdoor environments, as well as cameras that recover shape of objects with challenging material properties. These cameras are, for the first time, capable of measuring detailed ( $<100$ microns resolution) scans in extremely demanding scenarios with low-cost components. Several of these cameras are making a practical impact in industrial automation, being adopted in robotic inspection and assembly systems.

\section{BIOGRAPHY}

Mohit Gupta is an assistant professor in the Computer Science department at the University of Wisconsin-Madison. Earlier, he was a research scientist in the CAVE lab at Columbia University. He received a B.Tech. in computer science from Indian Institute of Technology Delhi, and a Ph.D. from the Robotics Institute, Carnegie Mellon University. His research interests are broadly in computer vision and computational imaging. His focus is on designing computational cameras that enable computer vision systems to perform 
robustly in extreme real-world scenarios, as well as capture novel kinds of information about the physical world that is not possible with conventional cameras. 